\title{
UMA ANÁLISE BIBLIOMÉTRICA SOBRE REUSO DO CONHECIMENTO NO DESENVOLVIMENTO DE PRODUTO
}

Fabio do Carmo (fcarmoracing@hotmail.com) - PPGEM, Universidade Tecnológica Federal do Paraná

Juliana Schmidt (jschmidt@alunos.utfpr.edu.br) - PPGEM, Universidade Tecnológica Federal do Paraná

Milton Borsato (borsato@utfpr.edu.br) - PPGEM, Universidade Tecnológica Federal do Paraná

\section{RESUMO}

O processo de desenvolvimento de produto (PDP) é composto por várias atividades de engenharia, tais como design, simulação, cálculo e outras. Algumas dessas atividades podem ser repetitivas e demoradas, retratando uma dificuldade em indústrias cada vez mais exigentes. Uma maneira de diminuir a repetitividade e reduzir o tempo e os investimentos nesses processos é a introdução de técnicas de reuso do conhecimento no PDP. Segundo La Rocca (2011), estas técnicas, no entanto, por anos têm estado concentradas em apenas algumas indústrias mais avançadas, como a automotiva e a aeroespacial. Objetivando difundir técnicas de reuso do conhecimento no PDP a outras indústrias, retratando as principais tendências de pesquisa e o que falta ser pesquisado, este trabalho apresenta um procedimento de seleção de artigos, compreendendo publicações entre 2012 e 2017, a fim de compor um referencial bibliográfico relevante sobre o assunto. A partir de mais de 3000 artigos, a pesquisa identificou os 37 mais relevantes, a partir de bases de dados internacionais. Realizou-se também uma análise bibliométrica dessas pesquisas, descrevendo quais trabalhos, autores e periódicos foram os mais relevantes. Ao final, uma análise sistêmica foi conduzida identificando as principais propostas, os problemas que elas atacam, e as oportunidades de pesquisa mencionadas.

Palavras-chave: Reuso do conhecimento; desenvolvimento de produto; PDP; bibliometria; análise sistêmica

Área: Organização e Gestão do Conhecimento para o Desenvolvimento de Produtos

\section{INTRODUÇÃO}

A Indústria 4.0 é considerada a quarta revolução industrial, sendo possibilitada pelo surgimento da Manufatura Inteligente. $O$ conceito de Indústria 4.0 consiste em unir tecnologias industriais, de comunicação e de informação a fim de formar uma manufatura não apenas automatizada, mas também inteligente e customizável. O objetivo da Indústria 4.0 é de ser altamente flexível ao ponto de o consumidor fazer um pedido de um produto, a fábrica recebê-lo, e iniciar sua produção automaticamente, realizando inclusive o envio deste produto ao consumidor no final do processo (ZHOU; LIU; ZHOU, 2015).

De acordo com Kang et al. (2016), enquanto o "lean manufacturing" procurava eliminar desperdícios em custo, durante os anos 80 e 90, a Manufatura Inteligente visa gerenciar e melhorar os principais fatores existentes na manufatura, como produtividade, 
qualidade, entrega e flexibilidade. Segundo Helu et al. (2015), a Manufatura Inteligente combina capacidade avançada de manufatura e tecnologias digitais em todas as fases da vida do produto, para endereçar problemas como aumento de competitividade, aumento da demanda de maior variedade de produtos, e diminuição da disponibilidade de mão de obra especializada na manufatura.

Todos esses conceitos que possibilitam a Indústria 4.0, não podem compor um ciclo de processos completamente "inteligente" se a fase de projeto de produto ainda compreender tarefas repetitivas e demoradas. É incoerente possuir um "chão de fábrica" completamente automatizado e flexível, se os projetistas de produto ainda perdem muito tempo procurando informações (e.g. de projetos passados para utilizar em atividades atuais). Tais tarefas não agregam valor ao produto final, pois são provenientes de conhecimento adquirido em projetos anteriores, e este conhecimento deveria ser rapidamente recuperado e empregado de uma forma mais automatizada, não sendo necessário refazer a mesma tarefa várias vezes a cada novo projeto. Em muitas indústrias, porém, este tipo de rotina é uma realidade, onde o projetista acaba por executar a mesma atividade inúmeras vezes.

As técnicas de reuso do conhecimento no PDP atacam este problema. Segundo Verhagen et al. (2012) e Chapman e Pinfold (2001), estas técnicas compreendem um grupo de soluções que auxiliam as atividades de engenharia em diversas etapas do processo de desenvolvimento de produto, a fim de automatizá-las e customizá-las. Este fim se dá pela capacidade de tais métodos em recuperar conhecimentos adquiridos em projetos anteriores e empregá-los nos projetos atuais. La Rocca (2011) afirma que estas técnicas, no entanto, por anos têm estado concentradas em apenas algumas indústrias mais avançadas tecnologicamente, como a automotiva e a aeroespacial.

A fim de retratar as atuais tendências acerca das pesquisas sobre o tema reuso do conhecimento no PDP, identificar as lacunas existentes e tornar estas técnicas mais acessíveis a outras indústrias e pesquisadores, o presente trabalho reúne, através de um processo científico, os principais artigos que tratam do assunto. $\mathrm{O}$ procedimento de seleção de artigos adotado foi o ProKnow-C, dos autores Ensslin et al. (2010), que visa auxiliar o pesquisador a encontrar os trabalhos mais relevantes para um determinado tema. Após a seleção dos artigos, foi realizada uma análise bibliométrica, a qual, segundo Ensslin et al. (2010), é baseada em métodos de avaliação quantitativa a fim de identificar quais são os trabalhos, autores, periódicos e palavras-chave mais relevantes para um determinado assunto. Na sequência uma análise sistêmica é apresentada, investigando o conteúdo desses artigos. A presente pesquisa é dividida em três fases principais: A seleção do Portfólio Bibliográfico, a Análise Bibliométrica e a Análise Sistêmica desse portfólio. Desta forma, este trabalho é dividido em seis seções: introdução, o processo de seleção de portfólio bibliográfico, a análise bibliométrica, a análise sistêmica, as conclusões e as referências utilizadas na pesquisa.

\section{SELEÇÃO DO PORTFÓLIO BIBLIOGRÁFICO}

A sequência adotada, com base na ferramenta ProKnow-C, para a seleção do Portfólio Bibliográfico foi a seguinte:

Figura 1. Sequência para seleção de artigos. Fonte: dos autores

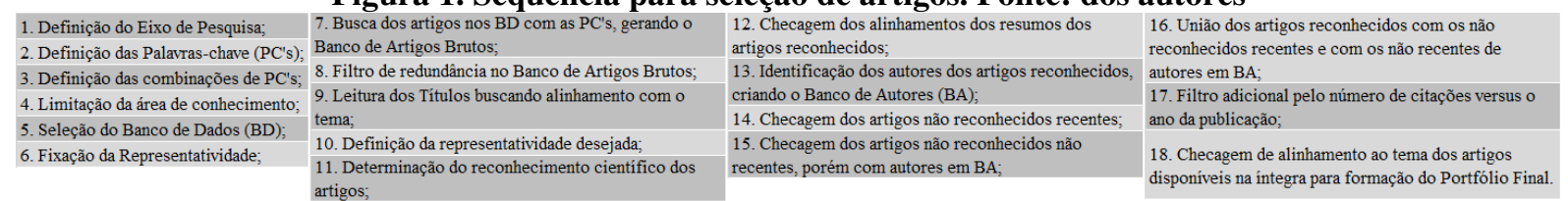


A seleção inicia-se com a definição do eixo de pesquisa. Com o intuito de obter um grande número inicial de artigos, decide-se realizar as buscas com palavras na língua inglesa, não limitando assim a pesquisa por artigos escritos somente em português. O eixo de pesquisa definido é o "Knowledge in Product Development", e as palavras-chave (PC's) usadas foram:

\begin{tabular}{|c|c|}
$\begin{array}{c}\text { Figura 2. Combinações das palavras-chave. Fonte: dos autores } \\
\text { knowledge reuse } \\
\text { information reuse } \\
\text { Knowledge capture } \\
\text { product information exchange } \\
\text { Knowledge representation } \\
\text { ontologies }\end{array}$ & $\begin{array}{c}\text { product development } \\
\text { digital manufacturing }\end{array}$ \\
\hline $\begin{array}{c}\text { knowledge management } \\
\text { design reuse }\end{array}$ & collaborative product development \\
KBE & New Product Development \\
\hline \begin{tabular}{c} 
NPD \\
\hline design knowledge management \\
knowledge visualization
\end{tabular} & product design \\
\hline $\begin{array}{c}\text { collaborative design } \\
\text { engineering design }\end{array}$ \\
\hline
\end{tabular}

O primeiro grupo, composto por 11 PC's, foi combinado com o segundo grupo composto por 10 PC's, gerando 110 combinações diferentes através do produto resultante da multiplicação entre os dois grupos. A área de conhecimento selecionada foi engineering, e os bancos de dados escolhidos para as pesquisas foram o Web of Science, Scopus, Springer Link, IEEE, Emerald e Compendex. A representatividade fixada foi o de retorno de um único artigo relacionado ao tema para manter o banco de dados (BD's). Todos os BD's foram mantidos. Em seguida iniciou-se as buscas nos BD's com as combinações de PC's. Essa fase deu-se entre o dia 16 de março de 2017 até o dia 31 de março de 2017. O primeiro grupo de PC's foi combinado ao segundo grupo no campo de busca através do operador lógico "AND”, mantendo os dois termos entre aspas, conforme o exemplo: "design reuse" AND "product development". Utilizou-se filtros de artigos publicados a partir de 2012, com os tipos de documentos sendo artigos de periódicos ou de congresso. As PC's foram pesquisadas primeiro nos títulos, depois nos resumos e em seguida nas palavras-chave, obtendo-se as seguintes quantidades de artigos para cada banco de dados: Web of Science (43), Scopus (2431), Springer Link (70), Emerald (126), IEEE (95) e Compendex (326). Os 3091 artigos encontrados compõem o chamado Banco de Artigos Brutos Preliminar. A seguir realizou-se um filtro de redundância, excluindo os artigos repetidos e não escritos em língua inglesa. $\mathrm{O}$ Banco de Artigos Brutos passou a conter então 1201 trabalhos. Através da leitura dos títulos, verificou-se quais estavam alinhados ao assunto. Apenas 696 artigos demonstraram satisfazer tal condição.

A fim de definir a representatividade desejada, foi levantado o número de citações de cada um dos artigos. Definiu-se que a representatividade desejada para o grupo de artigos que comporiam o portfólio principal seria de $40 \%$ das citações acumuladas, a fim de se chegar a um portfólio final com não mais que 40 artigos. Isto equivale a 20 citações ou mais. Desta forma, 28 artigos possuíam reconhecimento científico, compondo assim o Repositório K, enquanto os demais, i.e. 668 artigos, ainda sem reconhecimento cientifico confirmado, o Repositório P. Através da leitura dos resumos do Repositório K, verifica-se quais dos 28 artigos estão alinhados ao tema, o que resulta na seleção de 18 artigos. Estes compõem o Repositório A. Segue-se com a identificação dos autores deste repositório, a fim de criar o Banco de Autores (BA). Foram identificados 68 nomes. Em seguida fez-se a seguinte verificação: dos artigos do Repositório $\mathrm{P}$, foram verificados os recentes (menos de 2 anos). Caso se encontrasse nestas condições, lia-se o resumo para verificar se estava alinhado ao tema. Em caso positivo o artigo passava para o Repositório B. Caso contrário, o artigo era eliminado. Para os artigos que não são recentes, verificou-se se os autores estavam no Banco de Autores. Em caso positivo lia-se o resumo. Caso este estivesse alinhado ao tema deveria passar para o Repositório B, caso contrário, era eliminado. Quando os autores não estavam no 
Banco de Autores o artigo era imediatamente eliminado. O resultado desta verificação foi que 168 artigos do Repositório P foram aprovados ao Repositório B. Estes foram então fundidos aos artigos do Repositório A, para formar o Repositório C.

Identificou-se, entretanto, a necessidade de aplicar um filtro adicional ao Repositório C, a fim de conseguir um Portfólio Final com não mais de 40 artigos, visto que um número exagerado de trabalhos na seleção final poderia tornar mais difícil a sua consulta. Decidiu-se então eliminar artigos provenientes do Repositório B, através do critério da tabela abaixo.

\begin{tabular}{|c|c|}
\hline \multicolumn{2}{|c|}{ Tabela 1. Critério de filtragem } \\
\hline ANO DA PUBLICAÇÃo & QTDE. DE CITAÇões MENOR QUE \\
\hline 2016 & 2 \\
\hline 2015 & 3 \\
\hline 2014 & 5 \\
\hline 2013 & 7 \\
\hline 2012 & 9 \\
\hline
\end{tabular}

Este filtro adicional resultou em 130 eliminações, deixando 38 artigos provenientes do Repositório B selecionados. O Repositório C passou a ter 56 artigos. Verificou-se então se os artigos do Repositório $\mathrm{C}$ estavam disponíveis na íntegra, através do acesso à plataforma Capes, e se estavam alinhados ao assunto. Foram encontrados 37 artigos disponíveis e alinhados, sendo que o restante foi eliminado. Estes trabalhos encontrados na íntegra compõem, portanto, o Portfólio Final.

Tabela 2. Portfólio Final

\begin{tabular}{|c|c|c|c|c|}
\hline Cód. & TíTULO DO ARTIGO & ANO & AUTORES & $N^{\circ}$ DE CITAÇÕES \\
\hline 1 & The evolution, challenges, and future of knowledge representation in product design system & 2013 & Chandrasegaran, Senthil K. (1); & 297 \\
\hline 2 & A framework for design knowledge management and reuse for Product-Service Systems in c & c 2012 & Zhang, D., Hu, D., Xu, Y., Zhang & 52 \\
\hline 6 & A flexible assembly retrieval approach for model reuse & 2012 & Chen, X., Gao, S., Guo, S., Bai, . & 41 \\
\hline 10 & A semantic product modeling framework and its application to behavior evaluation & 2012 & Lee, J.H., Fenves, S.J., Bock, C. & 26 \\
\hline 14 & Implementation of knowledge-based engineering methodology in ship structural design & 2012 & Yang, H.Z., Chen, J.F., Ma, N., I & 25 \\
\hline 17 & A framework for management of Knowledge-Based Engineering applications as software se & 2012 & Bermell-Garcia, P., Verhagen, ' & 24 \\
\hline 18 & A semantic representation model for design rationale of products & 2013 & Zhang, Y., Luo, X., Li, J., Buis, J. & 24 \\
\hline 25 & Automatically generating assembly tolerance types with an ontology-based approach & 2013 & Zhong, Y., Qin, Y., Huang, M., L & 22 \\
\hline 28 & A design of knowledge management tool for supporting product development & 2013 & Zhen, L; Wang, L; Li, JG & 20 \\
\hline 32 & A personalized query expansion approach for engineering document retrieval & 2014 & Hahm, G.J., Yi, M.Y., Lee, J.H., & 17 \\
\hline 46 & A framework for optimising product performance through feedback and reuse of in-service & 2015 & Igba, J., Alemzadeh, K., Gibbon & 14 \\
\hline 59 & What ontologies for PLM: A critical analysis & 2016 & Mostefai, S., Bouras, A. & 12 \\
\hline 64 & Knowledge evaluation in product lifecycle design and support & 2014 & Xu, Y., Bernard, A., Perry, N., X & 11 \\
\hline 79 & Enriching the semantics of variational geometric constraint data with ontology & 2015 & Lu, W., Qin, Y., Liu, X., Huang, I & 9 \\
\hline 97 & A formal ontology-based spatiotemporal mereotopology for integrated product design and & 2015 & Gruhier, E., Demoly, F., Dutartı & 7 \\
\hline 100 & Constructing a meta-model for assembly tolerance types with a description logic based app & 2014 & Zhong, Y., Qin, Y., Huang, M., L & 7 \\
\hline 114 & An organizational approach to designing an intelligent knowledge-based system: Applicatior & 2015 & Girodon, J., Monticolo, D., Bor & 6 \\
\hline 149 & Semantic relation based personalized ranking approach for engineering document retrieval & 2015 & Hahm, G.J., Lee, J.H., Suh, H.W & 5 \\
\hline 139 & Design and implementation of an integrated surface texture information system for design, & 2014 & Qi, Q., Scott, P.J., Jiang, X., Lu, & 5 \\
\hline 156 & Description logic-based automatic generation of geometric tolerance zones & 2015 & Qin, Y., Lu, W., Liu, X., Huang, I & 4 \\
\hline 160 & Domain knowledge versioning and aggregation mechanisms in product design processes & 2015 & Zouari, A., Tollenaere, M., Ben & 4 \\
\hline 164 & Heuristics-based design process & 2016 & Calle-Escobar, M., Mejía-Gutié & 4 \\
\hline 178 & Using prototypes to leverage knowledge in product development: Examples from the auton & 2016 & Erichsen, J.A.B., Pedersen, A.L. & 4 \\
\hline 215 & Semantic-based knowledge categorization and organization for product design enterprises & 2015 & Liu, X.-J., Wang, Y.-L. & 3 \\
\hline 186 & BOM-based design knowledge representation and reasoning for collaborative product deve & 2016 & Peng, Gongzhuang (1); Mao, $\mathrm{H}$ & 3 \\
\hline 238 & Antecedents of Knowledge Sourcing and Reuse from a Knowledge Repository in the Virtual I & | 2016 & Filieri, R., Willison, R. & 2 \\
\hline 246 & Contribution for Analysing, Saving and Prioritising of Lessons Learned Issues Regarding Prod & 2016 & Bracke, S., Inoue, M., Ulutas, E & 2 \\
\hline 271 & Ontological approach for organisational knowledge re-use in product developing environme & $\epsilon 2016$ & Cheung, W.M., Maropoulos, P. & 2 \\
\hline 221 & A Design Knowledge Representation and Reuse Method Based on Ontology and Knowledge & 2016 & Y. Yan; J. X. Yang; J. Hao; G. X. & 2 \\
\hline 223 & A framework to capture and share knowledge using storytelling and video sharing in global $f$ & 2016 & Zammit, J.P., Gao, J., Evans, R. & 2 \\
\hline 242 & Capturing and Sharing Product Development Knowledge Using Storytelling and Video Sharin! & 2016 & Zammit, J., Gao, J., Evans, R. & 2 \\
\hline 263 & Knowledge Sourcing and Knowledge Reuse in the Virtual Product Prototyping: An Explorator & 2016 & Filieri, R., Alguezaui, S. & 2 \\
\hline 383 & Product design and manufacturing process based ontology for manufacturing knowledge re & 2017 & Peter ChhimRatna Babu Chinn & 1 \\
\hline 299 & A RFBSE model for capturing engineers' useful knowledge and experience during the design & 2017 & Qin, H., Wang, H., Johnson, A.l & 1 \\
\hline 442 & A sketch-based semantic retrieval approach for 3D CAD models & 2017 & Qin, F.-W., Gao, S.-M., Yang, X & 0 \\
\hline 467 & Anisotropic diffusion map based spectral embedding for 3D CAD model retrieval & 2017 & Lin, Xin (1, 2); Zhu, Kunpeng (2) & 0 \\
\hline 615 & Reuse-oriented common structure discovery in assembly models & 2017 & Wang, P., Zhang, J., Li, Y., Yu, J. & 0 \\
\hline
\end{tabular}




\section{ANÁLISE BIBLIOMÉTRICA}

Terminada a seleção dos artigos do Portfólio Final, iniciou-se então a análise bilbiométrica. Quatro aspectos foram avaliados: grau de relevância dos periódicos, reconhecimento científico dos artigos, participação dos autores no portfólio e as palavraschave mais utilizadas.

Quanto ao grau de relevância dos periódicos, o destaque ficou com o Computer-Aided Design, contendo 7 artigos do portfólio em suas publicações. O artigo mais reconhecido cientificamente foi o de código "1" (Tabela 2) com 297 citações. O autor com maior número de publicações no portfólio foi Wenlong Lu, com 5 publicações. Já a palavra-chave mais utilizada foi "knowledge management", com 9 utilizações.

Figura 3. Relevância dos periódicos. Fonte: dos autores

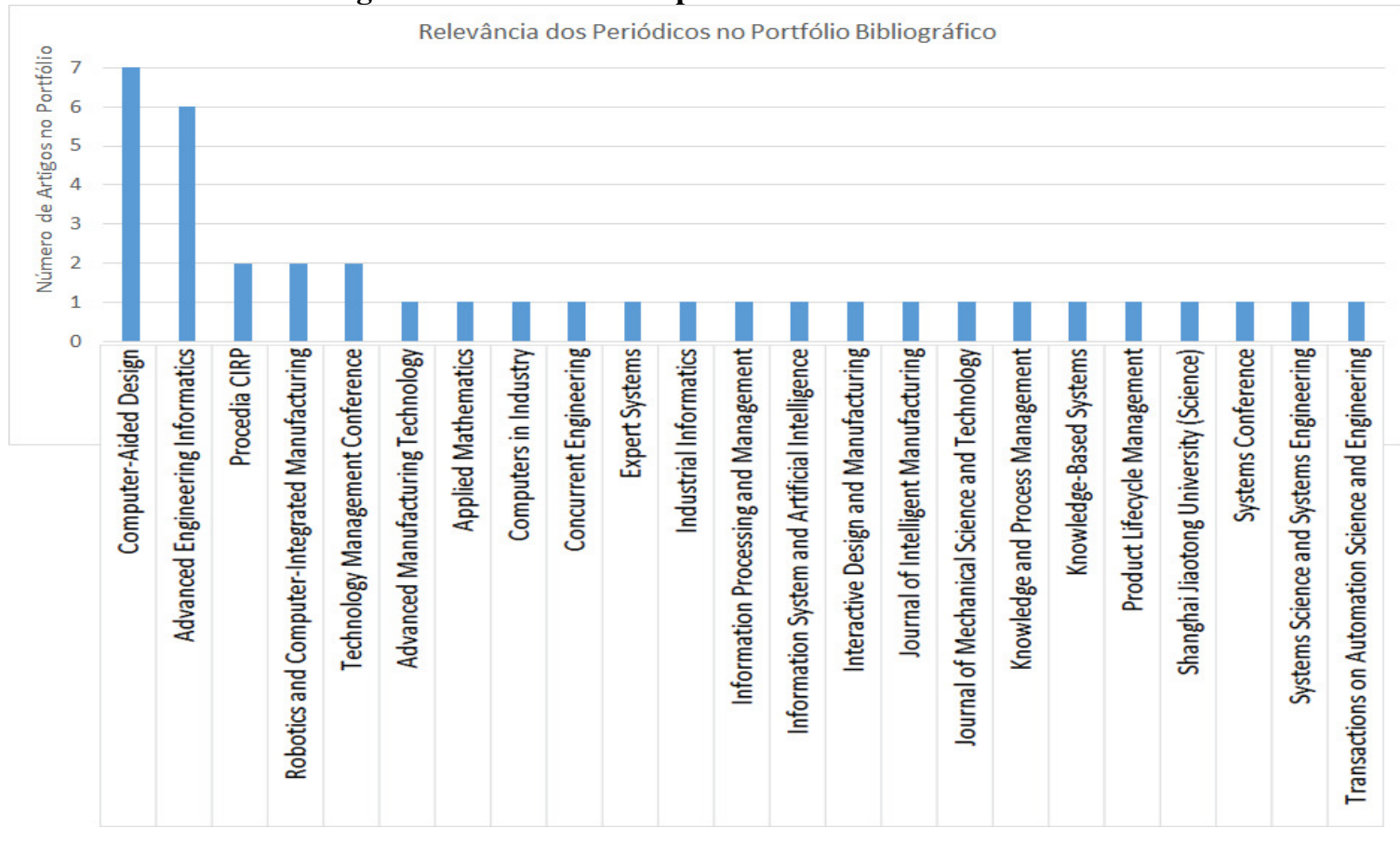

Figura 4. Relevância dos artigos. Fonte: dos autores

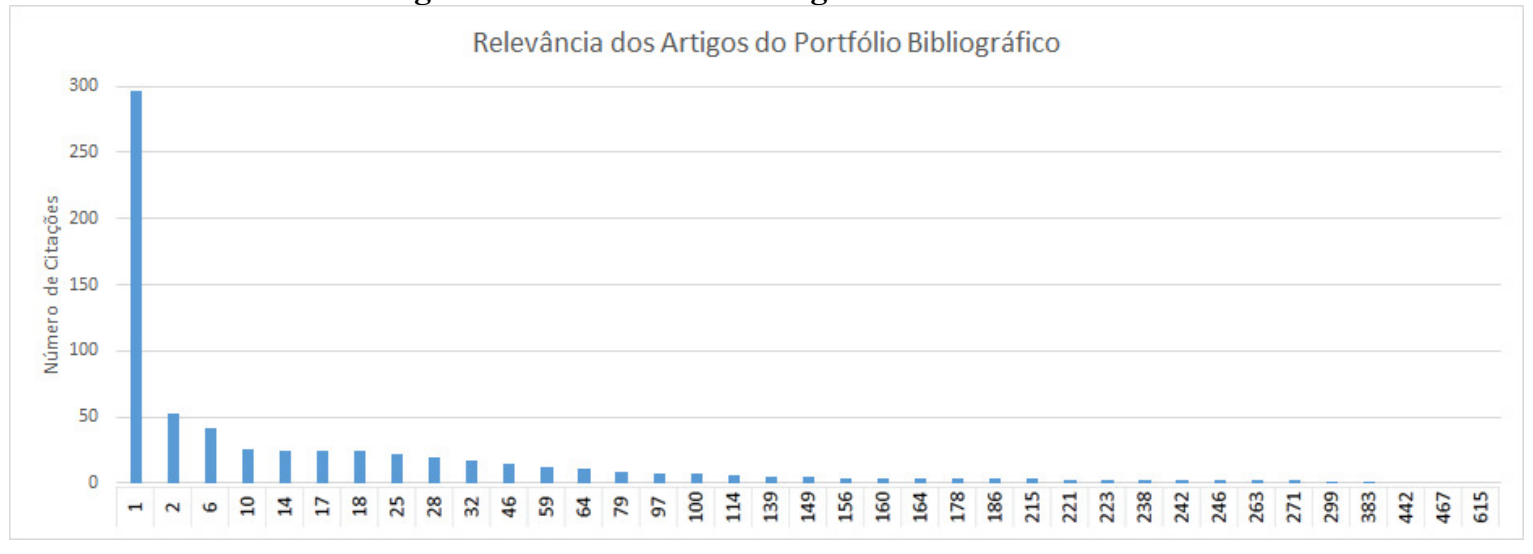


Figura 5. Autores com maior participação. Fonte: dos autores

Autores com maior participação no PB

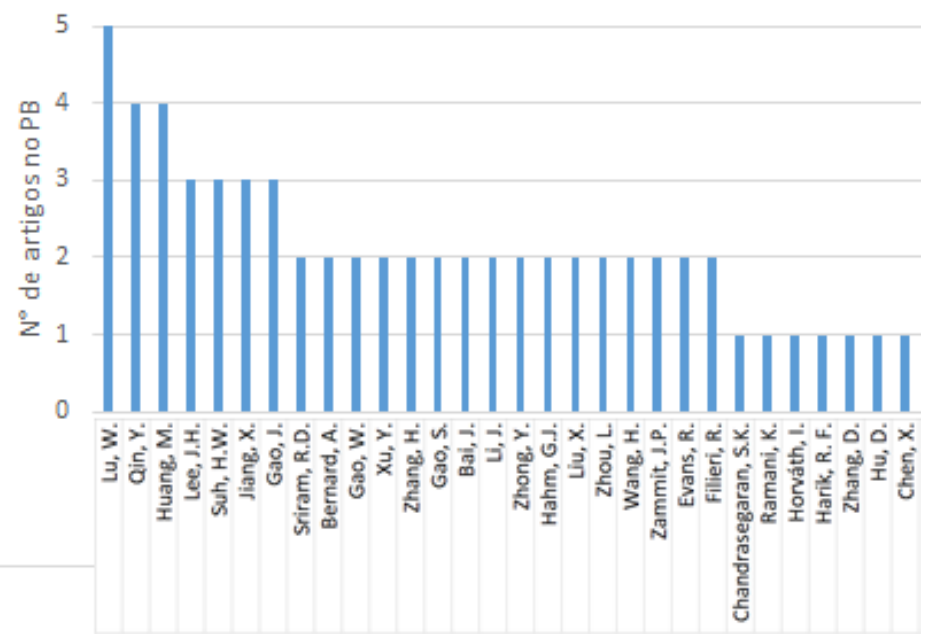

Figura 6. Palavras-chave mais utilizadas. Fonte: dos autores

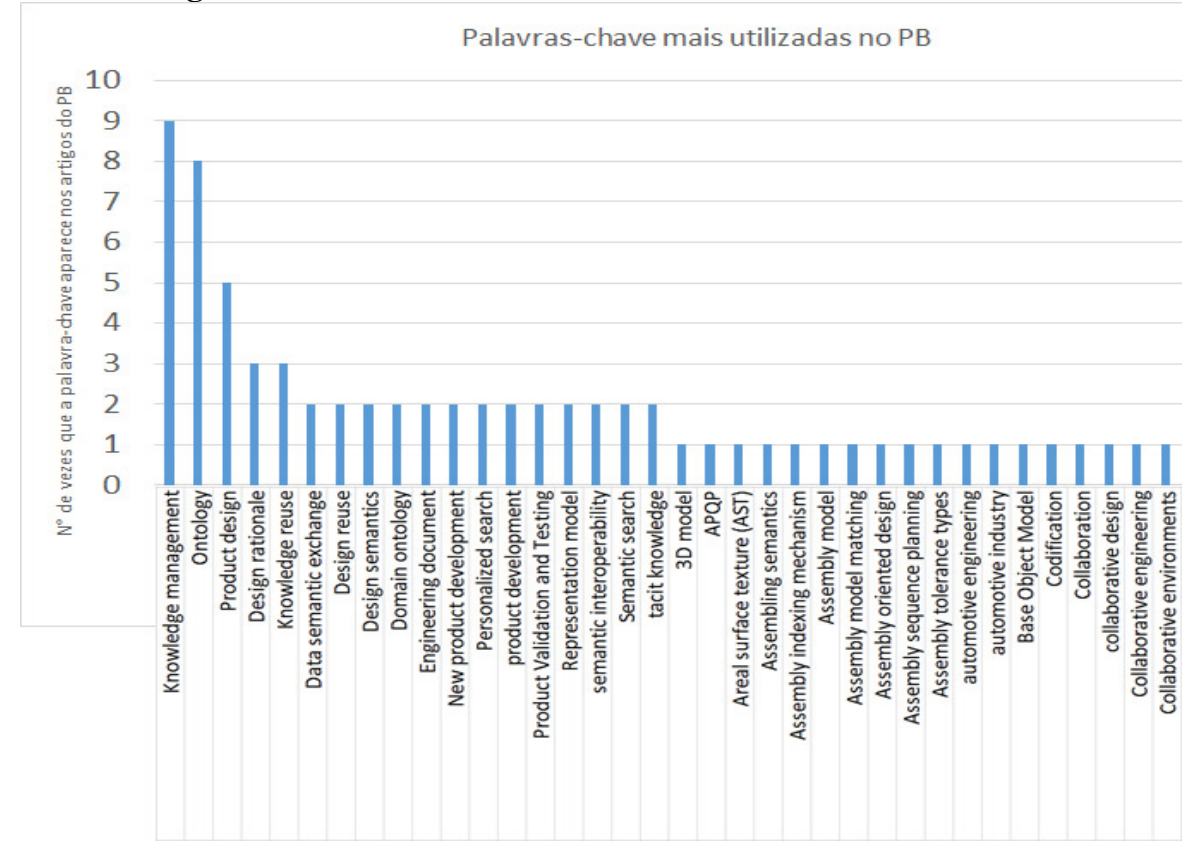

\section{ANÁLISE SISTÊMICA}

Esta fase analisou o conteúdo dos trabalhos selecionados no Portfólio Final, a fim de listar os problemas identificados nos trabalhos, os objetivos propostos para solucionar esses problemas e as lacunas existentes mencionadas. Os trabalhos foram agrupados em categorias para cada um desses 3 temas (problema, objetivo e lacuna), através da similaridade entre seus conteúdos. Os artigos são referenciados pelos seus códigos, conforme a Tabela 2.

\subsection{Problemas identificados}

Foram constatados 7 grupos de problemas mencionados nos trabalhos, listados a seguir. 
Revisão de literatura: Os artigos de código "1" e " 215 " compreendem este grupo. O primeiro apresenta a necessidade de revisão do tema representação do conhecimento no PDP, enquanto o último identifica o problema de haver uma diferença de objetivos entre pesquisas de conhecimento na engenharia e pesquisas sobre gerenciamento do conhecimento.

Problemas de representação semântica: Este grupo compreende seis trabalhos, “10", “97”, “32", “149”, "79" e "59". Os quatro primeiros expõem os problemas de precisão na representação semântica de produtos e documentos, reduzindo a confiabilidade na recuperação de informações passadas. O quinto trabalho aponta a falta de semântica nos dados de geometria de produto, enquanto último identifica a necessidade de encontrar a melhor semântica para trabalhar com sistemas PLM.

Relacionados a tolerâncias dimensionais e geométricas: Com três artigos, "25", "156" e "100", este grupo trata das dificuldades em se representar, gerar, e transferir dados de tolerâncias. Os dois primeiros indicam a necessidade de haver correta especificação de tolerâncias em modelos de montagem, enquanto o último trata da impossibilidade de transferências de dados semânticos de tolerâncias com os padrões atuais de sistemas.

Problemas relacionados a CAD: Grupo composto por seis artigos. O trabalho "139" aponta as limitações de ferramentas atuais para medição de texturas em superfícies geradas por CAD. "442" identifica a imprecisão na recuperação de características baseadas em esboços. "467" enxerga a necessidade de encontrar uma nova maneira de recuperar modelos CAD. "615" trata da necessidade em identificar similaridade entre modelos de montagem. " 6 " fala sobre a falta de maturidade na recuperação de modelos de montagem, e "14" identifica a necessidade de integrar desenho inteligente ao PDP.

Problemas no gerenciamento do conhecimento: Este é o maior grupo, compreendendo dez artigos. As pesquisas " 2 " e " 238 ", identificam a necessidade de gerenciar conhecimento dentro do ambiente PDP. O trabalho "17" aponta para a necessidade de personalizar e codificar o conhecimento no PDP. De acordo com "18" existe uma necessidade em haver informações de lógica de desenho (Design Rationale) no PDP. "114" alega falta de pesquisa em sistemas KBE (Knowledge Based Engineering) que levem em conta todos os mecanismos do conhecimento. Já "160" trabalha com o problema de se rastrear as versões das informações. "164" identifica a necessidade de fixação, reutilização e de tornar tangível o conhecimento no PDP. "246" trata da necessidade em se aprender com lições passadas, enquanto " 271 " fala da necessidade em se utilizar ontologias para gerenciar o conhecimento no PDP. Por fim, "64", trata da demanda por questões de conhecimento no PDP.

Problemas na captura e transferência de conhecimento: Esta categoria é representada em oito artigos. O trabalho " 221 " aponta a falta de eficiência na reutilização do conhecimento de desenho. "186" identifica a necessidade de transferir conhecimento eficientemente, enquanto " 28 " enxerga a necessidade em se facilitar o compartilhamento da aquisição de conhecimento no PDP. Segundo " 223 " e " 242 ", existe dificuldade em capturar e transmitir conhecimento dentro do ambiente de PDP, e " 263 " retrata as dificuldades no reuso do conhecimento gerado no PDP. "46" indica a dificuldade da transmissão do conhecimento de campo a fim de aumentar a vida do produto, e "383" identifica uma falta de pesquisas sobre ontologias levando conhecimento de manufatura ao PDP.

Problemas individuais: Este último grupo de artigos não apresenta similaridade entre nenhuma das categorias anteriores. É composto por "178" e "299". O primeiro afirma que há necessidade em discutir o poder de melhorar conhecimento implícito no PDP através de protótipo, enquanto o último identifica uma falta de pesquisas sobre integração entre conhecimento de produto e conhecimento de resolução de problemas. 


\subsection{Entregas propostas}

Nove categorias de entregas foram identificadas entre os artigos do Portfólio Final.

Revisão de literatura: Apenas o artigo " 1 ” entrega uma revisão da literatura, neste caso sobre representação do conhecimento no PDP.

Automatização de tarefas CAD: Este tipo de entrega é proposta por seis trabalhos. O artigo "6" propõe uma abordagem para recuperação de modelos de montagem, enquanto "442" aborda recuperação semântica baseada em sketches para modelos 3D. "467" também propõe uma abordagem para recuperação de modelos CAD, e "615" indica um método para identificar similaridades entre modelos de montagem. A proposta de "139" é de um sistema integrado de informações de textura de superfícies, enquanto "14" descreve uma ferramenta de reuso do conhecimento no PDP de embarcações, que compreende modelagem automática de componentes.

Automatização de tolerâncias: Três artigos propõem este tipo de entrega. $\mathrm{O}$ artigo " $25 "$ descreve uma abordagem para geração automática de tolerâncias, enquanto o "100" produz outra abordagem para construção de modelos de montagem com tolerâncias possuindo descrição lógica. O trabalho "156" é apenas extensão de uma pesquisa anterior dos mesmos autores.

Uso de conhecimento de outros processos: Esta categoria é composta pelos artigos "46", "97" e "383". O primeiro trabalho propõe um framework para gerenciamento de conhecimento de campo. As duas últimas pesquisas entregam uma proposta de ontologia para utilização de conhecimento de manufatura dentro do PDP.

Soluções para avaliações: Três trabalhos pertencem a este grupo. O artigo "64" desenvolve um método de avaliação do conhecimento. O trabalho "59" propõe um método para avaliação de ontologias para PLM, enquanto a pesquisa " 238 " descreve um modelo de avaliação de sistemas de gerenciamento do conhecimento.

Representação do conhecimento: Este grupo compreende seis artigos. A pesquisa "79" tem como proposta desenvolver abordagem para melhoria de semântica nos dados de produto. Já o artigo "18" propõe um modelo baseado em ontologia para representação semântica de informações de lógica de desenho (Design Rationale). O artigo "10" desenvolve um framework para modelagem semântica de produto. " 215 " apresenta um modelo de representação do conhecimento e modelo de ontologia, enquanto "242" traz um framework para reconhecimento dirigido pelo usuário e pesquisa sobre utilização de vídeos para captura do conhecimento. O último trabalho deste grupo, "299", trata de um modelo para representação de conhecimento e experiência do projetista no PDP.

Ferramenta para gerenciar conhecimento: Este é o maior grupo, com nove artigos. Os trabalhos "28", "2", “32", “160" e "186" possuem propostas muito similares de entregas. Todos eles desenvolvem frameworks para gerenciamento geral do conhecimento no PDP. $\mathrm{O}$ artigo " 271 " entrega uma técnica baseada em ontologia para gerenciamento do conhecimento no PDP. "17" propõe um framework para gerenciamento de sistemas KBE, enquanto "149" traz uma abordagem para classificação de documentos de engenharia. O último trabalho deste grupo, "246", propõe uma abordagem para gerenciamento de lições aprendidas no PDP.

Transferência de conhecimento: Esta categoria é composta por quatro trabalhos. O artigo " 178 " entrega um framework para uso de protótipo a fim de transferir conhecimento. A pesquisa " 221 " apresenta um método para reuso do conhecimento de desenho, enquanto "223" traz um framework para melhorar compartilhamento de conhecimento tácito. O artigo “263” desenvolve um estudo sobre as barreiras ao reuso do conhecimento no PDP. 
Temas individuais: Dois artigos propõem entregas que não se encaixam em nenhuma das categorias anteriores: "114" e "164". O primeiro apresenta uma metodologia para projetar um sistema KBE inteligente, enquanto o último propõe uma abordagem para introdução de heurística no PDP.

\subsection{Lacunas identificadas}

Foram identificadas 7 categorias de sugestões para trabalhos futuros. Alguns trabalhos se encaixam em múltiplas categorias, devido a diferentes sugestões de trabalhos apresentadas num mesmo artigo.

Sociais e sustentáveis: Dois artigos sugerem trabalhos futuros relacionados a esta categoria, "1" e "263". O primeiro propõe o desenvolvimento de plataformas de gerenciamento do conhecimento globais sustentáveis, enquanto o último sugere testar as influências de condições ambientais no reuso do conhecimento no PDP.

Melhoria da entrega proposta: A maior parte dos artigos propõe melhorias em suas próprias entregas propostas. É o caso dos artigos "6", "10", "97", "28", "2", “32", "64", "156", “139”, “149”, “160”, “186”, “114”, “221”, “383”, “442” e "615”. Destaque para o trabalho "64", que sugere integração da entrega proposta com sistemas CAD comerciais, e para o artigo "139", que propõe criar liberdade para usuários avançados atualizarem o sistema estudado.

Criação de entregas similares: A pesquisa " 25 " sugere a criação de outras ferramentas de interpretação de tolerâncias, enquanto o trabalho "139" propõe a criação de sistema similar, porém mais simples, para usuários menos avançados.

Avaliação financeira da entrega proposta: Dois artigos compõe este grupo, os trabalhos " 46 " e "17". O primeiro sugere avaliar o benefício financeiro em implantar a entrega proposta, enquanto o último propõe medir os custos e benefícios da solução.

Criar entregas diferentes da proposta: Três artigos se encaixam nesta categoria, as pesquisas "64", "32" e "1". O primeiro dá três sugestões para trabalhos futuros. Desenvolver método para otimização do peso de produto, desenvolver método para PDP dinâmico e desenvolver método para sistemas de PDP paralelos e distribuídos. O segundo trabalho dá duas sugestões. Desenvolver métodos para avaliação e atualização de ontologias, e avaliar a eficácia da captura de mudanças de interesse do usuário. O último trabalho sugere desenvolver um framework de arquitetura aberta para gerenciamento do conhecimento no PDP.

Realizar extensão do estudo feito: Assim como na categoria de melhorias das entregas propostas, este grupo também reuniu vários artigos, listados a seguir: "59", "32", “149”, “160”, “238”, “79”, “164”, “246”, “178”, “223”, “17”, “263”, “18”, “100”, “2”, “186” e "467". Destaque para sugestões de extensão do estudo para outros domínios "32", "149", "160" e "238", e sugestões de outras formas de avaliar e/ou validar as entregas propostas “238”, “164”, "246” e "263”.

Não mencionam lacunas: Os seguintes trabalhos deixam de identificar oportunidades de pesquisas futuras: "14", “215”, "242”, "271" e "299”.

\section{CONCLUSÃO}

O presente artigo apresenta os procedimentos para a seleção de um portfólio com os artigos mais relevantes sobre o tema reuso do conhecimento no PDP. Para tal, realizou-se uma análise bibliométrica para identificar os principais trabalhos, autores e periódicos que 
publicaram sobre o tema, além de uma análise sistêmica, que permitiu agrupar os artigos que compõem o Portfólio Final em categorias de problemas, entregas e lacunas.

No início da pesquisa, 3091 artigos foram encontrados. A partir da sequência proposta pelo método ProKnow-C, se chegou a um portfólio de 37 trabalhos. Este foi submetido a uma análise bibliométrica, onde destacou-se as contribuições do artigo "1", com o maior número de citações.

Através da análise sistêmica foi possível verificar várias oportunidades de pesquisa, como a melhoria dos objetos propostos através de sua integração com outros sistemas, e as sugestões de validar algumas das soluções propostas de forma distinta das originais.

A presente pesquisa, no entanto, teve algumas restrições de tempo para sua realização. Não foi possível por exemplo executar a terceira fase do processo ProKnow-C, que consiste em realizar um teste de representatividade do portfólio criado, através do levantamento de citações das referências de todos os artigos do portfólio, para posterior filtragem e inserção na seleção atual. Esta seria uma oportunidade de pesquisa futura. Outra lacuna existente foi o filtro extra que foi aplicado após a elaboração do Repositório $\mathrm{C}$, para reduzir o número de artigos reunidos. Seria interessante seguir com o estudo, a partir deste ponto, removendo este filtro, e verificar se os artigos extras trazem algum tema não explorado.

As tendências e lacunas identificadas neste trabalho, podem servir de referência para estudos posteriores, auxiliando o pesquisador no entendimento de quais são as pesquisas mais relevantes na área, e do que ainda não foi pesquisado.

\section{REFERÊNCIAS}

CHAPMAN, C.B.; PINFOLD, M. The application of a knowledge based engineering approach to the rapid design and analysis of an automotive structure. Advances in Engineering Software, v32, p.903-912, 2001.

ENSSLIN, L.; ENSSLIN, S.; LACERDA, R.; TASCA, J. ProKnow-C, knowledge development process - constructivist. Processo técnico com patente de registro pendente junto ao INPI. 2010a.

HELU, M.; MORRIS, K.; JUNG, K.; LYONS, K.; LEONG, S. Identifying performance assurance challenges for smart manufacturing. Manufacturing Letters. Gaithersburg, Maryland, Estados Unidos da América, v6, p.1-4, 2015.

KANG, H.S.; LEE, J.Y.; CHOI, S.; KIM, H.; PARK, J.H.; SON, J.Y.; KIM, B.H.; NOH, S.D. Smart Manufacturing: Past research, present findings, and future directions. International Journal of Precision Engineering and Manufacturing-Green Technology, v3, n.1, p.111-128, 2016.

LA ROCCA, G. Knowledge based engineering techniques to support aircraft design and optimization. TU Delft, Delft University of Technology, 2011.

VERHAGEN, W.J.C.; BERMELL-GARCIA, P.; VAN DIJK, R.E.C.; CURRAN, R. A critical review of Knowledge-Based Engineering: An identification of research challenges. Advanced Engineering Informatics, v26, p.5-15, 2012.

ZHOU, K.; LIU, T.; ZHOU, L. Industry 4.0: Towards future industrial opportunities and challenges. 12th International Conference on Fuzzy Systems and Knowledge Discovery (FSKD), 15-17 de Agosto, 2015. Zhangjiajie, China. 